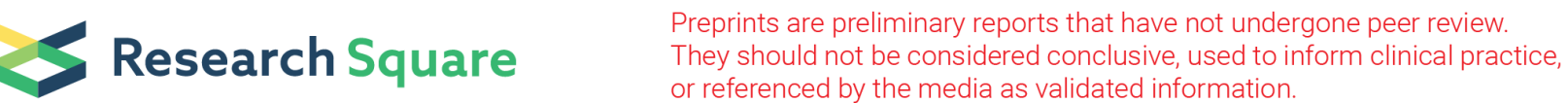

\section{Whole genome analysis and characterization of Salmonella enterica subsp. enterica serovar Typhimurium strain UPM 260 for mediated genetic manipulation}

Najwa Syahirah Roslan

Universiti Putra Malaysia

Nurulfiza Mat Isa ( $\nabla$ nurulfiza@upm.edu.my)

Universiti Putra Malaysia https://orcid.org/0000-0002-4726-7728

Abdul Rahman Omar

Universiti Putra Malaysia

Mohd. Hair Bejo

Universiti Putra Malaysia

Aini Ideris

Universiti Putra Malaysia

\section{Research article}

Keywords: Salmonella Typhimurium, genetic manipulation, virulence factor, whole genome sequencing (WGS), whole genome annotation

Posted Date: January 3rd, 2020

DOI: https://doi.org/10.21203/rs.2.20015/v1

License: (c) (i) This work is licensed under a Creative Commons Attribution 4.0 International License.

Read Full License 


\section{Abstract}

Background Salmonella enterica serovar Typhimurium persists as one of the most frequent food-borne zoonoses, causing a major public health concern worldwide. Furthermore, Salmonella infection has a large economic impact. Globally, the main sources of infection for humans include the consumption of contaminated poultry meat and eggs. In animals however, Salmonella transmission usually occurs horizontally from infected birds and contaminated environments. Hence, to delve further on how the impact of this disease can be lessened, an epidemiological study needs to be performed. It is vital to determine the genomic sequences of microorganisms to understand their biology and functional characterization. Thus, we determined the whole-genome sequence and virulence profile of S. enterica serovar Typhimurium strain UPM 260 isolated from Perak, Malaysia. Whole genome sequencing (WGS) using paired-end sequencing generated 107 contigs with a total genome size of $4.9 \mathrm{Mbp}$ and $52 \% \mathrm{G}+\mathrm{C}$ content. The contigs were annotated for phylogenetic and functional analysis.

Results Through the analysis, it is revealed that the genome were resistant to a number of antimicrobial drug classes including aminoglycoside, fluoroquinolone, tetracycline and phenicol. Also found in UPM 260 genome were three intact prophages (Fels-1, Gifsy-2 and one unique prophage, mEp390). The genome housed four types of restriction-modification systems (RMS) and Type I-E subtype of CRISPRCas system. Two metal resistance operons (mer and cop) and six pathogenicity islands (SPIs) were also discovered in UPM 260 genome. The SPIs contributed mostly to the bacterial virulence properties since 1054 CDS were reported to be homologous to the virulence factors in the database VFDB.

Conclusion This study benefits us specifically in the field of genome engineering where gene-based genetic manipulations can be applied in reducing the prevalence and pathogenicity in Salmonella.

\section{Background}

Salmonella enterica has been regarded as one of the most recurring food-borne pathogens isolated from humans and animals alike. Outbreaks in developing countries have caused a huge economic burden, particularly on the surveillance, prevention and treatments (Eng et. al., 2015). Food animal industries, mainly poultry, are the most susceptible since the abattoirs are known to be the main reservoir of Salmonella contamination. In United States of America alone, there are more than a million Salmonella outbreaks reported, with almost 600 deaths each year (Leekitcharoenphon et. al, 2016). The prevalence of salmonellosis in Malaysia, albeit rarely apparent, has doubled in recent years. In 2014, a study was done to isolate Salmonella from poultry farms in Malaysia where $11.9 \%$ out of 12664 samples isolated were identified as Salmonella (Ong et. al., 2014). Interestingly, the number of Salmonella isolates were notably low at farm level suggesting good farm practices were being implemented. However, a high level of cross contamination was observed during transportation all the way through to slaughter and production process. 
Poultry has been the ultimate host for Salmonella colonization and was deemed asymptomatic most of the time. It is believed that the profound stress among the poultry during handling and transportation, mainly due to the overcrowded cages and long travelling hours, has triggered an increase in the shedding causing a serious case of lateral transmission. Presence of Salmonella isolates in the plastic cages during transportation has been well documented (Nidaullah et. al., 2017). Additionally, the constant high moisture in the slaughterhouse and wet market are thought to be an ideal niche for Salmonella to prosper and colonize by attaching to surfaces like plastics, cement and steels, where they prominently produced biofilms. All these factors eventually leading to Salmonella being transmitted into our food chains.

Salmonella enterica serovar Typhimurium is a facultative anaerobe, Gram-negative flagellated rodshaped bacterium with approximate size of 2-5 microns in length and 0.5-1.5 microns in diameter, it is one of the most frequently reported serovars along with $\mathrm{S}$. enterica serovar Enteriditis in food-borne outbreaks globally (Fabrega and Vila, 2013). S. Typhimurium caused the highest number of fatalities compared to other serovars (Enteritidis, Heidelberg, Newport and Montevideo) in the U.S from 2007 to 2011, with 9 deaths from 84 recorded cases (Andino and Hanning, 2015), with poultry, produce, and eggs as the main food vehicle attributing to the outbreaks. Since S. Typhimurium is not host-specific, it possesses a great threat and can occur everywhere either through ingestion of food and water contaminated with animals' waste, direct contact with infected animals or consumption of raw, undercooked meat.

Unlike S. Typhi, which induces typhoid fever, S. Typhimurium is mostly responsible for nontyphoidal salmonellosis (NTS) where its infections are often associated with gastroenteritis, with symptoms including non-bloody diarrhea, vomiting, nausea, headache, abdominal cramps and myalgias (Acheson and Hohmann, 2001). Symptoms of NTS can develop in just 6 to 12 hours upon ingestion and can last between 7 to 10 days. NTS infections can also lead to various complications including cholecystitis, pancreatitis and appendicitis (Crump et. al., 2008).

Of late, the surfacing of multidrug resistance (MDR) of NTS isolated from humans has caused a commotion in public health concerning the clinical management and relative prevention of the infection. Van et. al. (2007) conducted a surveillance study where five out of ninety-one Salmonella isolates were found to be multi-resistant (Albany, Anatum, Havana, London and Typhimurium). Unwarranted use of antibiotics for disease treatments and prophylaxis in the farm were proposed to be the root of the problem of MDR emergence (Hyeon et. al., 2011). Furthermore, the existence of MDR has created a significant impact on the use of antibiotics for treatments, since most isolates are often found to be resistant to quinolones and third generation cephalosphorins. Ultimately, the lack of new antimicrobial agents available for treating MDR-infected patients might lead to an increment of morbidity and mortality rates. Furthermore, an epidemiological study carried out by Travers and Barza in 2002 reported that MDR strains instigated severe and prolonged syndromes compared to susceptible strains, suggesting that MDR strains are more pervasive and virulent. 
The logical approach to put a halt to the widespread of MDR strains is to control the infectivity at the farm-level, hence lessening the outbreak. However, a Salmonella-free poultry farm entails excessive expenditure especially on feed, hygiene and management. The costs, together with the injudicious use of antibiotics, means that vaccination may be a suitable alternative. It has been shown that vaccinations in poultry has helped prevent intestinal and cecal colonization, resulting in reduced fecal shedding and minimized risk of vertical transmission and egg contamination (Gast, 2007). Live, attenuated vaccines have long been an ideal vaccine construct since it can elicit cell-mediated immune response while still maintaining its immunogenicity. An attenuated vaccine pivotal point is the various deletions subjected onto the bacterial essential virulence factors and mutations on metabolic enzymes encoding genes (Detmer and Glenting, 2006).

Salmonellae activity has been shown to be profoundly diverse in between serovars, dependent on environmental stresses including temperature differences, types of chemicals present, and nutritional conditions based on food sources available (Joerger et. al., 2009). However, upon successful invasion, they thrive in the host cell by facilitating the clustered virulence factors presence within its genome.

Multiple studies have been done on virulence mechanisms of S. Typhimurium in mice and cattle, but little has been recorded in chicken; the main vector of salmonellosis to man. Based on previous studies, there are a few notable genes that have roles as an advance guard in order to maintain bacterial survival in the infected host cells. Genes associated with outer membranes, metabolic and fimbrial formation are crucial for colonization, while lipopolysaccharides-biosynthesis related genes are vital for both colonization and systemic infections in the host (Morgan et. al., 2004). In addition, it has also been observed that flagellaassociated genes are necessary in the initial stages of host infection. However, there are more possibilities left to be discovered pertaining to the virulence factors, thus, here we present an in-depth study to identify the virulent determinants and their roles contributing to pervasiveness on the bacteria. Globally, In Malaysia, there are very few whole genome sequencing and analysis studies done on S. Typhimurium strains and very little information about the genome insight was revealed thus far.

In this study, we performed a whole genome sequencing on the strain S. Typhimurium UPM 260 in order to obtain a precise reference genome for identification of genomic features, virulence clusters, and multidrug resistance gene analysis. This study also benefits us specifically in the field of genome engineering where gene-based genetic manipulations can be applied in reducing the prevalence and pathogenicity in Salmonella.

\section{Methods And Materials}

\section{Strain Collection and Antimicrobial Susceptibility Testing}

Salmonella enterica subsp. enterica serovar Typhimurium strain UPM 260 was collected in 2014 from a broiler house located in Perak, Malaysia. Antimicrobial susceptibility testing was done on the strain by implementing the disc diffusion method and results obtained were measured following the standards and guidelines described by Clinical and Laboratory Standards Institute (CLSI, 2010) and European 
Committee on Antimicrobial Susceptibility Testing (EUCAST). The following discs (Oxoid, Basingstoke, UK) represented the antibiotic drugs used: Ampicillin (AMP-10), Cefotaxime (CTX-5), Nalidixic Acid (NA30), Polymyxin B (PB-300), Tetracycline (TE-10) and Trimethoprim/sulphamethoxazole (SXT-25). The strain then was subjected to whole genome sequencing.

\section{Whole Genome Sequencing, De Novo Assembly and Annotation}

The whole genome sequencing of $S$. Typhimurium UPM 260 was performed using paired-end sequencing ( $2 \times 250$ bp read length) on the Illumina HiSeq platform. The de novo assembly process performed using CLC Genomic Workbench version 7.5.1 (Qiagen, USA). The draft genome obtained from the assembly was queried using BLASTn to obtain reference genomes for the scaffolding process. Five strains; Salmonella enterica subsp. enterica serovar Typhimurium str. LT2 (Accession No.: NC_003197.2), Salmonella enterica subsp. enterica serovar Typhimurium str. CDC 2009K-2059 (Accession No.: CP014983.1), Salmonella enterica subsp. enterica serovar Typhimurium str. USDA-ARS-USMARC-1898 (Accession No.: CP014971.2), Salmonella enterica subsp. enterica serovar Typhimurium strain BL10 (Accession No.: CP024619.1) and Salmonella enterica subsp. enterica serovar Typhimurium strain CFSAN018746 (Accession No.: CP028199.1) were chosen as reference genomes for they have the closest sequence similarity with UPM 260. Contiguous sequences of $S$. Typhimurium UPM 260 were scaffolded and gap-filled using MeDuSa (Bosi et al., 2015) and GapBlaster (De Sá et al., 2016) respectively. The scaffolds were concatenated by the addition of NNNNN that acted as linker sequence to form a 'pseudogenome'. The draft genome of $S$. Typhimurium UPM 260 was annotated using RAST server (Aziz et al., 2008) which predicted protein-encoding sequences, rRNA and tRNA genes, genes functions and subsystems present in the genome. The annotated draft genome of $S$. Typhimurium UPM 260 has been deposited in the European Nucleotide Archive with accession number GCA_902153325.1.

\section{Sequence Analysis of the $S$. Typhimurium UPM 260}

The $S$. Typhimurium UPM 260 draft genome was queried against a curated database for restriction enzymes and related proteins; REBASE (Roberts et al., 2015) in search of restriction-modification sites. Presence of metal-resistance genes was predicted using the Antibacterial Biocide and Metal Resistance Genes Database (BacMet) (http://bacmet.biomedicine.gu.se/) (Pal et al., 2014). Additionally, CRISPR regions were investigated using CRISPRCasFinder (Couvin et al., 2018) where evidence level 1 implied a CRISPR region with one to three spacers while evidence level 2 to 4 were termed as true CRISPR. The presence of protospacers adjacent to CRISPR (Cas proteins) were identified using the same tool with 'Subtyping' level of precision. The draft genome was then was queried for plasmid regions using PlasmidFinder (Carattoli et al., 2014). Correspondingly, the identification was also carried out using PATRIC BLAST against the plasmid contig database for optimisation of results.

The detection of prophage sequences within the genome was executed using PHAge Search Tool Enhanced Release (PHASTER) (http://phaster.ca/) (Arndt et al., 2016; Zhou et al., 2011). The identified prophages were further subjected against the Virulence Factor Database (VFDB) in search of potential virulence genes existing within the prophage regions. The draft genome was queried against the 
Comprehensive Antibiotic Resistance Database (CARD) (Jia et al., 2017) (https://card.mcmaster.ca/) to uncover the acquired antimicrobial resistance genes. Subsequently, Salmonella pathogenicity islands (SPIs) were identified by utilizing SPIFinder (https://cge.cbs.dtu.dk/services/SPIFinder/). The predicted SPIs were searched for the presence of virulence factors such as bacterial pathogens, toxins and virulence genes against VFDB (Chen et al., 2005). Finally, virulence factors residing within the genome of S. Typhimurium UPM 260 were classified using VFDB (http://www.mgc.ac.cn/VFs/main.htm).

To proceed with the analysis, two $S$. enterica subsp. enterica Typhimurium genomes were chosen to understand the virulence mechanisms and pathogenicity of these organisms. One strain, the complete genome of $S$. enterica subsp. enterica Typhimurium LT2 (Accession number: NC_003197.2) is the most studied Salmonella Typhimurium strain while the other one, S. enterica serovar I 4, [5], 12:i:- (Accession number: PQNA00000000) was selected due to its geographical similarity.

\section{Results And Discussion}

\section{Genome sequence information}

The genome features of $S$. Typhimurium UPM 260, $S$. Typhimurium LT2 and $S$. I 4, [5], 12:i:- are summarized in Table 1. The draft genome of $S$. Typhimurium UPM 260 is estimated to be $4,869,864 \mathrm{bp}$, with $52.0 \%$ GC content and 4,893 coding sequences (CDSs). The circular representation of the genome is shown in Figure 1. A total of 49 tRNAs and two rRNAs comprising one small subunit ribosomal RNA and one large subunit ribosomal RNA were also detected in $S$. Typhimurium UPM 260.

Table 1: General genome characteristics of $S$. Typhimurium UPM 260, $S$. Typhimurium LT2 and $S$. Typhimurium I 4, [5], 12:i:-

\begin{tabular}{lccc}
\hline \multicolumn{1}{c}{ Features } & UPM 260 & LT2 & I 4, [5], 12:i:- \\
\hline Status & Draft & Complete & Draft \\
Genome size (bp) & $4,869,864$ bp & $4,857,432$ bp & $4,829,816$ bp \\
GC content (\%) & $52 \%$ & $53 \%$ & $52.1 \%$ \\
Contigs & 14 & - & 111 \\
Scaffolds & - & - & - \\
Total no. of CDS & 4,893 & 4,489 & 4,933 \\
Total no. of tRNA & 51 & 85 & 75 \\
Total no. of rRNA & 4 & 22 & 10 \\
References & This work & (McClelland et al., 2001) & (Ngoi et. al., 2018) \\
\hline
\end{tabular}




\section{Susceptibility to antimicrobial compounds and antimicrobial resistance-encoding genes}

$S$. Typhimurium UPM 260 was susceptible to ampicillin $(16 \mathrm{~mm})$, nalidixic acid $(18 \mathrm{~mm})$ and trimethoprim/sulphamethoxazole $(24 \mathrm{~mm}$ ) (Table 2). This isolate was also found to be resistant to polymyxin $\mathrm{B}(13 \mathrm{~mm})$ and tetracycline $(0 \mathrm{~mm})$. Whereas the strain shows intermediate sensitivity towards cefotaxime (29mm).

Table 2: Antimicrobial susceptibility testing of $S$. Typhimurium UPM 260

\begin{tabular}{|c|c|c|c|c|}
\hline \multirow[t]{2}{*}{ Antibiotic } & \multicolumn{3}{|c|}{$\begin{array}{l}\text { Zone diameter breakpoint } \\
(\mathrm{mm})\end{array}$} & \multirow[t]{2}{*}{ Results (mm) } \\
\hline & $\mathrm{S} \geq$ & I & $\mathrm{R} \leq$ & \\
\hline Ampicilin (amp) & 15 & 0 & 14 & 16(sensitive) \\
\hline Cefotaxime (ctx) & 30 & $24-29$ & 23 & 29(intermediate) \\
\hline Nalidixic acid (na) & 18 & 0 & 17 & 18(sensitive) \\
\hline Polymyxin B (pb) & 15 & 15 & 14 & 13(resistant) \\
\hline Tetracycline (te) & 24 & 0 & 23 & 0 (resistant) \\
\hline $\begin{array}{l}\text { Trimethoprim-sulphamethoxazole } \\
\text { (sxt) }\end{array}$ & 17 & $14-16$ & 13 & 24 (sensitive) \\
\hline
\end{tabular}

A total of 35 genes were predicted to confer resistance against different antibiotics categories. Additionally, UPM 260 contained antibiotic resistance-encoding genes related to multiple chromosomally encoded efflux pump mechanisms comprised of ATP-binding cassette (ABC), multidrug and toxic compound extrusion (MATE) transporters, major facilitator superfamily (MFS) and resistance-nodulation-division (RND).

All antibiotic resistance genes identified in UPM 260 were associated with resistance to fluoroquinolone, penicillin, macrolide, fosfomycin, peptide, aminocoumarin, aminoglycoside, monobactam, carbapenem, rifamycin, triclosan, glycylcycline, tetracycline, cephalosporin, phenicol, cephamycin, penems, carbapenem rhodamine, benzalkonium chloride, streptogramin and nitroimidazole. 
There are a few unique genes predicted in the UPM 260 genome that are absent from LT2 and I 4,[5].12:i:- including AdeF, APH(3')-Ia (aphA1), APH(3")-Ib (StrA), APH(6)-Id (StrB), as well as plasmid-encoded genes $Q n r S 1$ and $\operatorname{VgaC}$ (Table 3). All these unique genes were previously reported to confer resistance towards kanamycin, streptomycin (Heinzal et al., 1988), ciprofloxacin, tetracycline, trimethoprim (Sumrall et al., 2014) and streptogramin (Novotna \& Janata, 2006).

The emergence of multidrug-resistance bacteria has caused life-threatening problems in cancer chemotherapy and the treatment of bacterial infections. The multidrug transporters in bacteria are what trigger the resistance to drugs as they decrease cellular drug accumulation (Zgurskaya and Nikaido, 2000). RND drug transporter genes, acrAB, acrD, acrEF, or genes belonging to the MFS (emrB and mdfA), MATE (mdtK) and ABC (macAB) transporter families were all present in the UPM 260 genome.

Gram-negative bacteria are usually much more resistant than Gram-positive bacteria to a wide range of drugs due to the presence of outer membrane (OM) barrier (Vaara, 1993). RND pumps in Gram-negative bacteria are predominantly effective in generating resistance as they form a tripartite complex with the periplasmic proteins of the membrane fusion protein family and the OM channels. This complex then pumped out drugs directly to the external medium (Nikaido \& Takatsuka, 2009). One RND pump out of the abundance present in the UPM 260 genome is the multiple drug efflux pump AcrB, a pump that contributes to multiple drugs resistance and is essential for virulence in $S$. enterica serovar Typhimurium. This finding was supported by previous finding where AcrB was found to be related to both intrinsic and acquired multidrug resistance of Salmonella (Nikaido et. al., 1998).

Also present in Gram-negative bacteria is the lipopolysaccharide (LPS) that plays an imperative role for antibiotic resistance (Peterson et al., 1987). Ultimately, complete deletion of said efflux pump coupled with mutation of LPS could attenuate bacteria while simultaneously achieving maximal susceptibility to several drugs including oxacillin, nafcillin, and erythromycin (Stubbings et al., 2005). 


\section{Restriction-modification systems (RMS)}

A total of four restriction-modification systems (RMS) were determined in $S$. Typhimurium UPM 260; Type I, Type II, Type III and Type IV. S.StyUK1II; a specificity subunit and M.SenTFII; a methyltransferase are both Type I enzymes found in the strain. The Type I methyltransferase was predicted to cleave at the GAGNNNNNNRTAYG recognition motif. M.Sen641III and StyUK1IV are Type II and Type IIG methyltransferases respectively, where M.Sen641III has an ATGCAT recognition sequence. One Type III RMS; M.Sen22462III, a methyltransferase, was found in S. Typhimurium UPM 260. A Type IV restriction enzyme, StyLT2Mrr was also predicted and is recognized as a methyl-directed restriction enzyme with unknown cleavage site. UPM 260, LT2 and I 4,[5],12:i:- have the same Type I, Type II and Type IV RMS (Table 4). While I 4,[5],12:i:- was void from any Type III (R-M), both UPM 260 and LT2 have M.Sen22462III and M.StyUK1I as their Type III RMS respectively.

Bacterial evolution has caused them to adapt strategies to recognize and distinguish extraneous foreign DNA from self-DNA. Restriction-modification systems (RMS), operated by two enzymatic activities consist of either restriction endonuclease (REases) or methyltransferase (MTase) which functions involved cleaving the invading DNA and methylating the host respectively.

Table 4: Summary of restriction-modification systems (RMS) in S. Typhimurium UPM 260, LT2, and I 4,[5],12:i:- 


\begin{tabular}{|c|c|c|c|c|}
\hline $\begin{array}{c}\text { Restriction- } \\
\text { Modification } \\
\text { System }\end{array}$ & UPM 260 & LT2 & I 4,[5],12:i:- & Function \\
\hline \multirow{2}{*}{ Type I } & S.StyUK1II & S.StyUK1II & S.StyUK1II & Specificity subunit \\
\hline & M.SenTFII & M.SenTFII & M.SenTFII & Methyltransferase \\
\hline \multirow[t]{2}{*}{ Type II } & StyUK1IV & StyUK1IV & StyUK1IV & $\begin{array}{l}\text { Restriction } \\
\text { enzyme/methyltransferase }\end{array}$ \\
\hline & M.Sen641III & M.Sen641III & M.Sen641III & Methyltransferase \\
\hline Type III & M.Sen22462III & M.StyUK1I & - & Methyltransferase \\
\hline Type IV & StyLT2Mrr & StyLT2Mrr & StyLT2Mrr & $\begin{array}{l}\text { Methyl-directed } \\
\text { restriction enzyme }\end{array}$ \\
\hline
\end{tabular}

There has been no in depth study on M.Sen22462III, beside that it consists of both mod and res genes which allow the genome to function either modification (Mod) or restriction (Res) following site recognition (Mücke et. al., 2001). Whilst, StyLT2Mrr was primarily recognized as an enzyme conferring genotoxicity on heterologous expression of some exotic type II MTases in E. coli K12 (Ghosh et. al., 2014). One important finding has identified Mrr to be an elicitor towards high (hydrostatic) pressure (HP)-induced SOS response (Artsen and Michiels, 2005). It is believed that the StyLT2Mrr found in Salmonella genome aids in generating bacteria adaptability by increasing the genetic repertory of bacterial populations thus allowing the bacteria to rapidly adapt to their new environment (Cirz et. al., 2005).

\section{Antibacterial Biocides and Metals Resistance Genes}

The UPM 260 genome was found to have metal resistance genes associated to ammonium, arsenic, cadmium, cobalt, copper, chromium, magnesium, manganese, mercury, nickel, phosphate, tellurium and zinc. Through an elaborate study on metal resistance and its correlation with antibiotic resistance Pal et. al (2017) reported that resistance genes against toxic metals and metalloids predated the antibiotic era. Furthermore, the industrial revolution also contributed to the rapid increase in metal production in the environment. Likewise, the presence of metal resistance genes (MRGs) in bacteria was also premediated by protist predation, in which they exploited metal poisoning to inactivate and kill bacteria (Hao et al., 2017). As a result, bacteria had evolved a new detoxification strategy against 
metal in order to avoid lethality by protists. Out of the abundance of metals used, mercury resistance was particularly imperative as its transposons can amass various resistances thus making it a possible vector for co-resistance (Summers, 2002).

A gene cluster that is responsible for mercury resistance constituting merA/G/R was identified in UPM 260 genome. The merA gene constitutes the mer operon; an operon accountable for storing mobile genetic elements containing transposases and plasmids (Barkay et al., 2003). This finding suggests that mercury resistance in bacteria could be transferred via horizontal gene transfer (HGT) among various bacterial species. A study by McIntosh et al (2008) found that although the prevalence of a multi drug resistance (MDR) strain was initially caused by the unwarranted use of antibiotic, it is possible for nonantibiotic selective agents like heavy metals to be associated as potential carrier for antibiotic resistance due to both elements often being located adjacent to each other in the integrons. The genome also encoded a few proteins responsible for mercury resistance including merR and merP; a regulatory protein of merA operon and transport protein accordingly.

Additionally, a few genes associated with copper resistance were detected in the genome. Copper resistance is regulated mostly by cue and cop systems, where the first is the primary mechanism of copper efflux (Bondarczuk \& Piotrowska-Seget, 2013), while the latter is a plasmid-driven system found on chromosomes (Monchy et al., 2006). CueARO and CopADFRS operons work in-sync to detoxify copper from the system. UPM 260 was found to also be resistant towards silver. Interestingly, as suggested by Hao et. al. (2015), the bacterial genome can acquire silver resistance loci due to the presence of copper resistance for they are homologs (2015).

\section{CRISPR-Cas system}

The $S$. Typhimurium UPM 260 genome was predicted to contain one CRISPR region at the lowest evidence level and two CRISPR regions with evidence level 4 (Table 5). CRISPR 2 and CRISPR 3 comprised of 4 and 21 spacers respectively and both regions were found adjacent to CRISPR-associated helicase, Cas2, Cas1, Cas6, Cas5, Cas7, Cse2, Cse1 and 
Cas3. Out of 25 spacers found, 7 of them were classified as protospacers homologous to plasmid sequences and one spacer (Spacer 12) is homologous to phage sequence.

Table 5: CRISPR regions identified in S. Typhimurium UPM 260 genome

\begin{tabular}{lccccc}
\hline CRISPR Locus & Position & Start & End & No. of spacers & Evidence level \\
\hline CRISPR & Scaffold_1_1 & 2469729 & 2469838 & 1 & 1 \\
CRISPR & Scaffold_1_2 & 2680128 & 2680418 & 4 & 4 \\
Cas cluster & CAS-TypeIE & 2680515 & 2688974 & 8 & \\
\hline CRISPR & Scaffold_1_3 & 2696550 & 2697884 & 21 & 4 \\
\hline
\end{tabular}

UPM 260 has the smallest number of spacers compared to other strains, both LT2 and I 4, [5],12:i:- were reported to have three evidence 4 CRISPR locus 2,3,4 and 1,2,3 with 55 and 53 spacers respectively (Table 6). The CRISPR-Cas system; consisting of the CRISPR array and cas gene is a versatile system in bacteria that procures immunity against exogenous DNA (Karimi et. al., 2018). The spacers located within the array are responsible for conferring protection upon integration of foreign elements. It works by transcribing and processing the spacers into small non-coding interfering CRISPR RNAs (crRNAs) and formed a guide-RNA (gRNA) by fusing with trans-activating CRISPR RNA (tracrRNA). The gRNA will direct the Cas protein toward invading DNA for specific cleavage of homologous sequences (Barrangou and Marraffini, 2014). It is hypothesized that strains with a greater number of spacers were constantly exposed to phages and DNA invasion along the lineage of the strain (Shariat et. al., 2015). This finding suggests that UPM 260 might have lower exposure to phages. However, some spacers identified were found to be in the same position within all studied strains which, as proposed by Mohammed and Cormican (2015), showed consistency with the theory of common ancestors.

Table 6: Distribution of CRISPR loci found in different $S$. Typhimurium strains 


\begin{tabular}{lcll}
\hline Strain & $\begin{array}{c}\text { Total no. of spacers in CRISPR } \\
\text { loci }\end{array}$ & \multicolumn{1}{c}{$\begin{array}{c}\text { Spacers no. in } \\
\text { CRISPR-2 }\end{array}$} & \multicolumn{1}{c}{$\begin{array}{c}\text { Spacers no. in } \\
\text { CRISPR-3 }\end{array}$} \\
\hline UPM 260 & 25 & $4(2680128-2680418)$ & $21(2696550-$ \\
& & & $2697884)$ \\
LT2 & 55 & $25(2561070-$ & $17(2579183-$ \\
& & $2563051)$ & $2580251)$ \\
I 4, & 53 & $15(17810-18756)$ & $6(18830-19224)$ \\
{$[5], 12: 1:-$} & & & \\
\hline
\end{tabular}

\section{Plasmid and Prophage}

One plasmid replicon, IncR, was found in the $S$. Typhimurium UPM 260 genome. IncR plasmids are characterized to locate qnrS1; a gene that confers resistance towards aminoglycosides, chloramphenicol and tetracycline (García-Fernández et. al., 2008). However, the properties of the $250 \mathrm{bp}$ IncR replicon in the UPM 260 genome are still poorly studied and an initial query against BLAST revealed the replicon to be a fragment of the RepB family plasmid replication initiator protein. The fragment was analyzed for any potential plasmids and a short 9.3kb DNA sequence resembling an incomplete plasmid tig00000003 of Klebsiella pneumoniae strain AR_0115 was discovered in Scaffold 3 (7520 bp - $16846 \mathrm{bp}$ ). The query covered $70 \%$ of the $39 \mathrm{~kb}$ plasmid with $99.9 \%$ nucleotide identity. An extended analysis uncovered replication protein repB and the toxin-antitoxin addiction system that promotes plasmid stable maintenance (Rychlik et. al., 2006) residing inside the plasmid-associated genomic regions. An antibiotic resistance gene $\operatorname{vga} C$ was also characterized within the plasmid. $V g a C$ belongs to ABC-F subfamily protein that provides resistance towards streptogramin (Kadlec et. al., 2010).

A $4.5 \mathrm{~kb}$ fragment was also found in the same scaffold 3 ( $1 \mathrm{bp}-4543 \mathrm{bp}$ ) that bears resemblance to plasmid pSH-01 of Salmonella sp. A few genes were identified including $\operatorname{imp} A$, transposases and a DNA polymerase V (Pol V) subunit umuC. Both impA and umuC, are vital in circumventing DNA damage following mutational changes or UV radiation (Reuven et. al., 2009; Mints \& Fives-Taylor, 2000). S. Typhimurium has been studied to have a common 94-kb virulence plasmid, pSLT where both LT2 and I 4,[5],12:i:- were 
confirmed to have. However, the lack of an intact Typhimurium-specific pSLT plasmid in the UPM 260 genome is still undetermined.

Among the four functional prophages conserved in $S$. Typhimurium LT2 (Fels-1 and -2, Gifsy-1 and -2), two of them; Fels-1 (39.2 kb) and Gifsy-2 (59 kb) and one unique prophage namely mEp390 (47.9 kb) were identified in the UPM 260 genome while I 4,[5],12:i:harbors Fels-1, Gifsy-1 and Gifsy-2. The differences in prophages between UPM 260 and I 4, [5],12:i:- from LT2 could suggest both strains underwent different evolutionary pathways from their respective ancestors. Since prophages are mobile elements, their acquisition might be a crucial factor in developing strains with augmented virulence and pathogenicity (Ngoi et. al., 2018).

Moreover, it has been widely shown that prophages increase intracellular survival rate of the bacteria (Herrero-Fresno et. al., 2014). Fugueroa-Bossi et. al. (2001) found that most $S$. Typhimurium have conserved Gifsy-1 and Gifsy-2 prophages in the genome (2001). However, Gifsy-1 was not detected in the UPM 260 genome. The lack of conserved prophages in bacterial genomes may suggest the strain has undergone genomic degradation either by deletions, insertions or by manifestations of pseudogenes (Panzenhagen et. al., 2018). mEp390, Fels-1 and Gifsy-2 in UPM 260 encoded for multiple virulence genes including gtrA/B, grvA, sodCI, sseI/srfH and sspH1. Interestingly, Gifsy-2 harbored an antivirulence gene, grvA, where its null mutation, coupled with the presence of sodCI will render the strain more virulent (Ho and Slauch, 2001). However, deletion of the whole Gifsy-2 prophage is proven to reduce the strain virulence by more than 100 -fold due to the loss of periplasmic superoxide dismutase, SodCI, a protein that acts as protease resistance and tether onto peptidoglycan (PGN) cell wall to combat phagocytic superoxide (Krishnakumar et. al., 2007).

\section{Salmonella Pathogenicity Islands (SPIs) and Virulence Factors}

The complexity of Salmonella pathogenicity corelates with the presence of numerous protective as well as offensive virulence factors (Groisman and Ochman, 1997). Virulence factors contributing to adhesion, invasion and toxin genes are clustered in the chromosome 
are identified as "Salmonella pathogenicity islands" (SPI) (Santos et. al., 2003). Two of the most important SPIs in $S$. enterica are Salmonella Pathogenicity Island-1 (SPI-1) and Salmonella Pathogenicity Island-2 (SPI-2) which carry the effector proteins and facilitate the direct injection of effector proteins into the cytosol of eukaryotic cells. Both SPIs also encode for type III secretion systems (T3SS).

A total of 1054 (21.54\%) out of 4893 CDS annotated were found to be homologous to the virulence factors in the database VFDB. Among the virulence factors, 12 of them were identified as T3SSs encoding gene clusters, in which eight of them are T3SS-1 related, located in SPI-1 including hil, iac, inv, org, prg, sic, spa and spr. Four additional gene clusters were recognized as T3SS-2 related genes, which are encoded in SPI-2 (ssa, sSC, sse, ssr) (Figure 2). Additionally, 9 TTSS-1 translocated effectors encoding genes (avrA, $\operatorname{sip} A B C$, sop $A / B / D / E 2$ and $s p t P)$, as well as 11 T3SS-2 trans-located effectors encoding genes (pipB/B2, sifA/B, sseF/I/J/K1/K2/L, sspH2), were found within the UPM 260 genome. It has been suggested that type III secretion systems aid specifically in injecting effector proteins into host cell (Weening et. al. 2005). S. Typhimurium UPM260 was found to contain a further six pathogenicity islands, namely SPI-3, SPI-4, SPI-5, SPI-7, SPI-13, as well as SPI-14.

Gene clusters encoding fimbrial adherence in UPM 260 genome included bcf, csg, fim, lpf, $s a f, s t b, s t c, s t d, s t f, s t h, s t i, s t j$ and $t c f$. Seven of these adherence determinants (bcf, fim, $s a f, s t b, s t d, s t h, s t i)$ are conserved in most Salmonella serovars. These findings show that these genes are vital in colonizing the host cell (Yue et. al. 2012). The UPM 260 genome was also found to contain 52 flagella-encoding genes including cheA/A2/B/D/R/V/V3/W/Y/Z, flgABCDEFGHIJKLMN, flhABCDEFG, fliABCDEFGHIJKLMNOPQRST and motAB genes. Flagella are proven to increase the virulence of Salmonellae, whilst flagella monomers induce an intrinsic immune response to aid in bacterial clearance from the host (Franchi et al., 2006; Miao et al., 2006).

Despite years of in-depth studies on bacterial pathogenicity along with the existence of countless antimicrobial drugs, new infections are still thriving in approximately 300 million 
reported cases with over 10 million deaths worldwide every year (WHO, 2014). To counter this epidemic, it is rational to induce prophylactic protection by vaccination for it is economical, safe and proven to be highly effective. With the continuous emergence of infectious diseases, there is a desperate need of easy-to-handle and efficacious vaccines. The utilization of an attenuated Salmonella strain as a potential vaccine vector has been getting large attention over the past few decades mainly due to snowballing evidence that they induced better protective immune response against infection by different species of Salmonella in humans and animals compared to killed or subunit counterparts (Tennant and Levine, 2015). Additionally, the avirulent derivatives were discovered to be powerful in delivering heterologous antigens to the gut-associated lymphoid tissue (GALT) thereby stimulating secretory as well as cellular and humoral immune responses to those antigens (Pasetti et al., 2011).

To date, there have been many approaches in producing attenuated Salmonella strains by means of auxotrophic requirements, truncated lipopolysaccharide structure or hypersensitivity to host bactericidal activities, as all of these were deemed to be conditional for full fledge virulence in vivo, suggesting that survival within macrophages is vital for Salmonella. For example, the deletion of the auxotrophic gene aro $A$ has rendered $S$. Typhimurium to be avirulent due to the strain's inability to synthesize aromatic amino acids required for its growth (Felgner et al., 2016). Another instance is the mutation of asd, aspartate-semialdehyde dehydrogenase encoding-gene, which is responsible for the formation of diaminopimelic acid (DAP). DAP is a vital component for bacterial cell walls and its absence in vivo would leave the bacteria unable to replicate within the host, and thus quickly eradicated (Pascual et al., 2013). Aside from that, there is also a mutant constructed by altering the gene responsible for the synthesis of carbohydrates such as galE where the mutations hindered the production of normal lipopolysaccharide (LPS), hence reducing its virulence in vivo (Nnalue and Stocker, 1987).

In $S$. Typhimurium, there are 83 regulators presumed to play roles in virulence (Yoon et al., 2009) which were found to be abundant in the Salmonella pathogenicity island (SPI). The role of both SPI-1 and SPI-2 have been well documented in various studies. It is known that 
both SPIs aid in inducing gastroenteritis (Wallis et al., 1999) and systemic infections (M. A. Jones et al., 2007). Proteins found in SPI-1, SPI-5 and SopE were translocated by T3SS-1 into epithelial cell and interacted with host cell machinery. Once infected into the cell, SopE activated Rho GTPases. This activation gives rise to the rearrangement of the actin cytoskeleton and stimulates membrane ruffling to endorse internalization of bacteria into non-phagocytic cells (Friebel et al., 2001). Genetic modification of SPI-1 reduces the aptitude of $S$. Typhimurium from entering the intestinal epithelium, which correlates with its ability to provoke an inflammatory response (Santos et al., 2003).

Alteration of the ssaU gene, a major structural component gene located in SPI-2 has displayed considerable attenuation based on the bacterial numbers recovered from the liver of infected birds. It has been suggested that the mutation has inhibited the SPI-2 machinery production by hampering any production of effector proteins (Morgan et al., 2004). As a result, the complete function of the T3SS was completely abrogated thus making it attenuated.

Constructing an affectual vaccine candidate often required laborious screening for a suitable virulence gene to be mutated, and there are thousands of genes in a bacterial genome. There is also an impending risk of the mutation being inadequate in reducing the virulence. Besides the usual virulence determinants deletion, a serious consideration should also be placed towards the mutations of bacterial appendages. Bacterial appendages such as flagella, pili, needles, and capsules are located on the cell surface, and their expression is normally tightly controlled by the bacteria Pascual et al., 2013). Moreover, bacterial surface structures perform functions critical for pathogenesis and have evolved to withstand forces exerted by the external environment and cope with defenses mounted by the host immune system (Thanassi, Bliska and Christie, 2012).

$S$. Typhimurium induced membrane ruffling in two distinct cell types during its internalization into the intestinal epithelial cells; absorptive epithelial cells and $\mathrm{M}$ cells (Bäumler, Tsolis and Heffron, 1996). Bacterial invasion into M cells has inevitably caused an upsurge of bacterial attachments onto Peyer's patches due to an inclined tropism. It has 
been shown that IpfABCDE, an Typhimurium fimbrial operon, is largely involved in colonization of murine Peyer's patches. Attenuation of $l p f C$, a fimbrial outer membrane usher gene results in a 5 -fold increased $\mathrm{LD}_{50}$ of $S$. typhimurium in mice infected intragastrically. $L p f C$ mutants were also recovered in lower numbers from systemic sites.

\section{Conclusion}

To summarize, we characterized the genome of $S$. enterica subsp. enterica serovar Typhimurium strain UPM 260 through whole genome sequencing (WGS). Salmonellosis caused by $S$. Typhimurium persists as a significant global challenge. Given the polyphyletic nature of this serovar and its niche adaptation, it remains vital to analyze the genome repertoire especially its antimicrobial resistance genes and virulence factors. This characterization facilitates greatly in selection of genes for genetic manipulation. There is no restriction in ruling out genes for editing, as long as the genes play significant roles pertaining to virulence and pervasiveness. This study demonstrates the need for more $S$. Typhimurium strains isolated in Malaysia to be sequenced and documented to help in a deeper understanding of their virulence and pathogenicity.

\section{References}

Acheson, D., \& Hohmann, E. L. (2001). Nontyphoidal salmonellosis. Clinical Infectious Diseases, 32(2), 263-269.

Aertsen, A., \& Michiels, C. W. (2005). Mrr instigates the SOS response after high pressure stress in Escherichia coli. Molecular microbiology, 58(5), 1381-1391.

Amachawadi, R. G., Shelton, N. W., Shi, X., Vinasco, J., Dritz, S. S., Tokach, M. D., ... \& Nagaraja, T. G. (2011). Selection of fecal enterococci exhibiting tcrB-mediated copper resistance in pigs fed diets supplemented with copper. Appl. Environ. Microbiol., 77(16), 5597-5603.

Andino, A., \& Hanning, I. (2015). Salmonella enterica: survival, colonization, and virulence differences among serovars. The Scientific World Journal, 2015.

Arndt D., Grant JR., Marcu A., Sajed T., Pon A., Liang Y., Wishart DS. 2016. PHASTER: a better, faster version of the PHAST phage search tool. Nucleic acids research 44:W16-21. DOI: 10.1093/nar/gkw387.

Aziz, R. K., Bartels, D., Best, A. A., DeJongh, M., Disz, T., Edwards, R. A., ... \& Meyer, F. (2008). The RAST Server: rapid annotations using subsystems technology. BMC genomics, 9(1), 75.

Barkay, T., Miller, S.M., Summers, A.O., 2003. Bacterial mercury resistance from atoms to ecosystems. FEMS Microbiol. Rev. 27 (June (2-3)), 355-384. 
Barrangou, R., \& Marraffini, L. A. (2014). CRISPR-Cas systems: prokaryotes upgrade to adaptive immunity. Molecular cell, 54(2), 234-244.

Bäumler, A. J., Tsolis, R. M., \& Heffron, F. (1996). The Ipf fimbrial operon mediates adhesion of Salmonella typhimurium to murine Peyer's patches. Proceedings of the National Academy of Sciences, 93(1), 279283.

Bondarczuk, K., \& Piotrowska-Seget, Z. (2013). Molecular basis of active copper resistance mechanisms in Gram-negative bacteria. Cell biology and toxicology, 29(6), 397-405.

Bosi E., Donati B., Galardini M., Brunetti S., Sagot MF., Lió P., Crescenzi P., Fani R., Fondi M. 2015. MeDuSa: A multi-draft-based scaffolder. Bioinformatics 31:2443-2451. DOI:

10.1093/bioinformatics/btv171.

Campos, J., Cristino, L., Peixe, L., \& Antunes, P. (2016). MCR-1 in multidrug-resistant and copper-tolerant clinically relevant Salmonella 1, 4,[5], 12: i:-and S. Rissen clones in Portugal, 2011 to 2015. Eurosurveillance, 21(26), 30270.

Carattoli A., Zankari E., Garcia-Fernandez A., Larsen MV., Lund O., Villa L., Aarestrup FM., Hasman H. 2014. In Silico detection and typing of plasmids using plasmidfinder and plasmid multilocus sequence typing. Antimicrobial Agents and Chemotherapy 58:3895-3903. DOI: 10.1128/AAC.02412-14.

Cavaco, L. M., Hasman, H., Stegger, M., Andersen, P. S., Skov, R., Fluit, A. C., ... \& Aarestrup, F. M. (2010). Cloning and occurrence of czrC, a gene conferring cadmium and zinc resistance in methicillin-resistant Staphylococcus aureus CC398 isolates. Antimicrobial agents and chemotherapy, 54(9), 3605-3608.

Chen L., Yang J., Yu J., Yao Z., Sun L., Shen Y., Jin Q. 2005. VFDB: A reference database for bacterial virulence factors. Nucleic Acids Research 33:D325-D328. DOI: 10.1093/nar/gki008.

Cirz, R.T., Chin, J.K., Andes, D.R., Crecy-Lagard, V.D., Craig, W.A., Romesberg, F.E.(2005) Inhibition of mutation and combating the evolution of antibiotic resistance. PLOS Bio/ 3: 1024-1033.

CLSI (2010) Perfomance Standards for Antimicrobial Susceptibility Testing - Twentieth Informational Supplement CLSI Document M100-S20. Wayne, PA: Clinical and Laboratory Standards Institute.

Couvin D., Bernheim A., Toffano-Nioche C., Touchon M., Michalik J., Néron B., C Rocha EP., Vergnaud G., Gautheret D., Pourcel C. 2018. CRISPRCasFinder, an update of CRISRFinder, includes a portable version, enhanced performance and integrates search for Cas proteins. Nucleic acids research 46:1-6. DOI: 10.1093/nar/gky425.

Crump, J. A., Kretsinger, K., Gay, K., Hoekstra, R. M., Vugia, D. J., Hurd, S., ... \& Hanna, S. S. (2008). Clinical response and outcome of infection with Salmonella enterica serotype Typhi with decreased susceptibility to fluoroquinolones: a United States foodnet multicenter retrospective cohort study. Antimicrobial agents and chemotherapy, 52(4), 1278-1284. 
De Sá PHCG., Miranda F., Veras A., De Melo DM., Soares S., Pinheiro K., Guimarães L., Azevedo V., Silva A., Ramos RTJ. 2016. GapBlaster - A graphical gap filler for prokaryote genomes. PLoS ONE 11:e0155327. DOI: 10.1371/journal.pone.0155327.

Detmer, A., \& Glenting, J. (2006). Live bacterial vaccines-a review and identification of potential hazards. Microbial cell factories, 5(1), 23.

Eng, S. K., Pusparajah, P., Ab Mutalib, N. S., Ser, H. L., Chan, K. G., \& Lee, L. H. (2015). Salmonella: a review on pathogenesis, epidemiology and antibiotic resistance. Frontiers in Life Science, 8(3), 284-293.

Fàbrega, A., \& Vila, J. (2013). Salmonella enterica serovar Typhimurium skills to succeed in the host: virulence and regulation. Clinical microbiology reviews, 26(2), 308-341.

Fang, L., Li, X., Li, L., Li, S., Liao, X., Sun, J., \& Liu, Y. (2016). Co-spread of metal and antibiotic resistance within ST3-IncHI2 plasmids from E. coli isolates of food-producing animals. Scientific reports, 6, 25312.

Felgner, S., Frahm, M., Kocijancic, D., Rohde, M., Eckweiler, D., Bielecka, A., ... \& Häussler, S. (2016). aroAdeficient Salmonella enterica serovar Typhimurium is more than a metabolically attenuated mutant. MBio, 7(5), e01220-16.

Franchi, L., Amer, A., Body-Malapel, M., Kanneganti, T. D., Özören, N., Jagirdar, R., ... \& Grant, E. P. (2006). Cytosolic flagellin requires Ipaf for activation of caspase-1 and interleukin $1 \beta$ in salmonella-infected macrophages. Nature immunology, 7(6), 576.

Friebel, A., Ilchmann, H., Aepfelbacher, M., Ehrbar, K., Machleidt, W., \& Hardt, W. D. (2001). SopE and SopE2 from Salmonella typhimurium activate different sets of RhoGTPases of the host cell. Journal of Biological Chemistry, 276(36), 34035-34040.

García-Fernández, A., Fortini, D., Veldman, K., Mevius, D., \& Carattoli, A. (2008). Characterization of plasmids harbouring qnrS1, qnrB2 and qnrB19 genes in Salmonella. Journal of Antimicrobial Chemotherapy, 63(2), 274-281.

Gast, R. K. (2007). Serotype-specific and serotype-independent strategies for preharvest control of foodborne Salmonella in poultry. Avian diseases, 51(4), 817-828.

Ghosh, A., Passaris, I., Tesfazgi Mebrhatu, M., Rocha, S., Vanoirbeek, K., Hofkens, J., \& Aertsen, A. (2014). Cellular localization and dynamics of the Mrr type IV restriction endonuclease of Escherichia coli. Nucleic acids research, 42(6), 3908-3918.

Gilmour, M. W., Thomson, N. R., Sanders, M., Parkhill, J., \& Taylor, D. E. (2004). The complete nucleotide sequence of the resistance plasmid R478: defining the backbone components of incompatibility group $\mathrm{H}$ conjugative plasmids through comparative genomics. Plasmid, 52(3), 182-202. 
Groisman, E. A., \& Ochman, H. (1997). How Salmonella became a pathogen. Trends in microbiology, 5(9), 343-349.

Hao, X., Lüthje, F. L., Qin, Y., McDevitt, S. F., Lutay, N., Hobman, J. L., ... \& Zhu, Y. G. (2015). Survival in amoeba-a major selection pressure on the presence of bacterial copper and zinc resistance determinants? Identification of a "copper pathogenicity island". Applied microbiology and biotechnology, 99(14), 5817-5824.

Hao, X., Li, X., Pal, C., Hobman, J., Larsson, D. J., Saquib, Q., ... \& Rensing, C. (2017). Bacterial resistance to arsenic protects against protist killing. Biometals, 30(2), 307-311.

Hasman, H., \& Aarestrup, F. M. (2002). tcrB, a gene conferring transferable copper resistance in Enterococcus faecium: occurrence, transferability, and linkage to macrolide and glycopeptide resistance. Antimicrobial agents and chemotherapy, 46(5), 1410-1416.

Heinzel, P., Werbitzky, O., Distler, J., \& Piepersberg, W. (1988). A second streptomycin resistance gene from Streptomyces griseus codes for streptomycin-3 "-phosphotransferase. Archives of microbiology, 150(2), 184-192.

Herrero-Fresno, A., Leekitcharoenphon, P., Hendriksen, R. S., Olsen, J. E., \& Aarestrup, F. M. (2014). Analysis of the contribution of bacteriophage ST64B to in vitro virulence traits of Salmonella enterica serovar Typhimurium. Journal of medical microbiology, 63(3), 331-342.

Ho, T. D., \& Slauch, J. M. (2001). Characterization of grvA, an antivirulence gene on the Gifsy-2 phage in Salmonella enterica serovar Typhimurium. Journal of bacteriology, 183(2), 611-620.

Hong, K. H., \& Miller, V. L. (1998). Identification of a Novel Salmonellalnvasion Locus Homologous to Shigella ipgDE. Journal of Bacteriology, 180(7), 1793-1802.

Hyeon, J. Y., Chon, J. W., Hwang, I. G., Kwak, H. S., Kim, M. S., Kim, S. K., ... \& Seo, K. H. (2011). Prevalence, antibiotic resistance, and molecular characterization of Salmonella serovars in retail meat products. Journal of food protection, 74(1), 161-166.

Jia B., Raphenya AR., Alcock B., Waglechner N., Guo P., Tsang KK., Lago BA., Dave BM., Pereira S., Sharma AN., Doshi S., Courtot M., Lo R., Williams LE., Frye JG., Elsayegh T., Sardar D., Westman EL., Pawlowski AC., Johnson TA., Brinkman FSL., Wright GD., McArthur AG. 2017. CARD 2017: Expansion and modelcentric curation of the comprehensive antibiotic resistance database. Nucleic Acids Research 45:D566D573. DOI: 10.1093/nar/gkw1004.

Joerger, R. D., Sartori, C. A., \& Kniel, K. E. (2009). Comparison of genetic and physiological properties of Salmonella enterica isolates from chickens reveals one major difference between serovar Kentucky and other serovars: response to acid. Foodborne pathogens and disease, 6(4), 503-512. 
Jones, M. A., Hulme, S. D., Barrow, P. A., \& Wigley, P. (2007). The Salmonella pathogenicity island 1 and Salmonella pathogenicity island 2 type III secretion systems play a major role in pathogenesis of systemic disease and gastrointestinal tract colonization of Salmonella enterica serovar Typhimurium in the chicken. Avian Pathology, 36(3), 199-203.

Kadlec, K., Pomba, C. F., Couto, N., \& Schwarz, S. (2010). Small plasmids carrying vga (A) or vga (C) genes mediate resistance to lincosamides, pleuromutilins and streptogramin A antibiotics in methicillin-resistant Staphylococcus aureus ST398 from swine. Journal of antimicrobial chemotherapy, 65(12), 2692-2693.

Karimi, Z., Ahmadi, A., Najafi, A., \& Ranjbar, R. (2018). Bacterial CRISPR Regions: General Features and their Potential for Epidemiological Molecular Typing Studies. The open microbiology journal, 12, 59.

Klumpp, J., \& Fuchs, T. M. (2007). Identification of novel genes in genomic islands that contribute to Salmonella typhimurium replication in macrophages. Microbiology, 153(4), 1207-1220.

Krishnakumar, R., Kim, B., Mollo, E. A., Imlay, J. A., \& Slauch, J. M. (2007). Structural properties of periplasmic SodCl that correlate with virulence in Salmonella enterica serovar Typhimurium. Journal of bacteriology, 189(12), 4343-4352.

Lacroix, F.J., Cloeckaert, A., Grepinet, O., Pinault, C., Popoff, M.Y., Waxin, H., and Pardon, P. (1996)

Salmonella typhimurium acrB-like gene: identification and role in resistance to biliary salts and detergents and in murine infection. FEMS Microbiol Lett 135: 161-167.

Leekitcharoenphon, P., Hendriksen, R. S., Le Hello, S., Weill, F. X., Baggesen, D. L., Jun, S. R., ... \& Aarestrup, F. M. (2016). Global genomic epidemiology of Salmonella enterica serovar Typhimurium DT104. Appl. Environ. Microbiol., 82(8), 2516-2526.

McIntosh, D., Cunningham, M., Ji, B., Fekete, F. A., Parry, E. M., Clark, S. E., ... \& Beattie, M. (2008). Transferable, multiple antibiotic and mercury resistance in Atlantic Canadian isolates of Aeromonas salmonicida subsp. salmonicida is associated with carriage of an IncA/C plasmid similar to the Salmonella enterica plasmid pSN254. Journal of Antimicrobial Chemotherapy, 61(6), 1221-1228.

McClelland, M., Sanderson, K. E., Spieth, J., Clifton, S. W., Latreille, P., Courtney, L., ... \& Hou, S. (2001). Complete genome sequence of Salmonella enterica serovar Typhimurium LT2. Nature, 413(6858), 852.

Miao, E. A., Alpuche-Aranda, C. M., Dors, M., Clark, A. E., Bader, M. W., Miller, S. I., \& Aderem, A. (2006). Cytoplasmic flagellin activates caspase- 1 and secretion of interleukin $1 \beta$ via Ipaf. Nature immunology, 7(6), 569.

Mintz, K. P., \& Fives-Taylor, P. M. (2000). impA, a gene coding for an inner membrane protein, influences colonial morphology of Actinobacillus actinomycetemcomitans. Infection and immunity, 68(12), 65806586 . 
Mohammed, M., \& Cormican, M. (2015). Whole genome sequencing provides possible explanations for the difference in phage susceptibility among two Salmonella Typhimurium phage types (DT8 and DT30) associated with a single foodborne outbreak. BMC research notes, 8(1), 728.

Monchy, S., Benotmane, M. A., Wattiez, R., Van Aelst, S., Auquier, V., Borremans, B., ... \& Vallaeys, T. (2006). Transcriptomic and proteomic analyses of the pMOL30-encoded copper resistance in Cupriavidus metallidurans strain CH34. Microbiology, 152(6), 1765-1776.

Morgan, E., Campbell, J. D., Rowe, S. C., Bispham, J., Stevens, M. P., Bowen, A. J., ... \& Wallis, T. S. (2004). Identification of host-specific colonization factors of Salmonella enterica serovar Typhimurium. Molecular microbiology, 54(4), 994-1010.

Mücke, M., Reich, S., Möncke-Buchner, E., Reuter, M., \& Krüger, D. H. (2001). DNA cleavage by type III restriction-modification enzyme EcoP15I is independent of spacer distance between two head to head oriented recognition sites. Journal of molecular biology, 312(4), 687-698.

Ngoi, S. T., Yap, K. P., \& Thong, K. L. (2018). Genomic characterization of endemic Salmonella enterica serovar Typhimurium and Salmonella enterica serovar I 4,[5], 12: i:-isolated in Malaysia. Infection, Genetics and Evolution, 62, 109-121.

Nidaullah, H., Abirami, N., Shamila-Syuhada, A. K., Chuah, L. O., Nurul, H., Tan, T. P., ... \& Rusul, G. (2017). Prevalence of Salmonella in poultry processing environments in wet markets in Penang and Perlis, Malaysia. Veterinary world, 10(3), 286.

Nikaido, H., Basina, M., Nguyen, V. Y., \& Rosenberg, E. Y. (1998). Multidrug Efflux Pump AcrAB of Salmonella typhimuriumExcretes Only Those $\beta$-Lactam Antibiotics Containing Lipophilic Side Chains. Journal of Bacteriology, 180(17), 4686-4692.

Nikaido H, Takatsuka Y. Mechanisms of RND multidrug efflux pumps. Biochim Biophys Acta. 2009;1794:769-81.

Nishino, K., Latifi, T., \& Groisman, E. A. (2006). Virulence and drug resistance roles of multidrug efflux systems of Salmonella enterica serovar Typhimurium. Molecular microbiology, 59(1), 126-141.

Nnalue, N. A., \& Stocker, B. A. (1987). Test of the virulence and live-vaccine efficacy of auxotrophic and galE derivatives of Salmonella choleraesuis. Infection and immunity, 55(4), 955-962.

Novotna, G., \& Janata, J. (2006). A new evolutionary variant of the streptogramin A resistance protein, Vga (A) LC, from Staphylococcus haemolyticus with shifted substrate specificity towards lincosamides. Antimicrobial agents and chemotherapy, 50(12), 4070-4076.

Ong, L. P., Muniandy, K., How, S. P., Yip, L. S., \& Lim, B. K. (2014). Salmonella isolation from poultry farms in Malaysia from 2011 to 2013. ETERINARY RESEARCH, 180. 
Pal, C., Bengtsson-Palme, J., Rensing, C., Kristiansson, E., Larsson, DGJ. (2014) BacMet: antibacterial biocide and metal resistance genes database, Nucleic Acids Res., 42, D737-D743. doi:

$10.1093 /$ nar/gkt1252

Pal, C., Asiani, K., Arya, S., Rensing, C., Stekel, D. J., Larsson, D. J., \& Hobman, J. L. (2017). Metal resistance and its association with antibiotic resistance. In Advances in microbial physiology (Vol. 70, pp. 261-313). Academic Press.

Panzenhagen, P. H. N., Cabral, C. C., Suffys, P. N., Franco, R. M., Rodrigues, D. P., \& Conte-Junior, C. A. (2018). Comparative genome analysis and characterization of the Salmonella Typhimurium strain CCRJ _26 isolated from swine carcasses using whole-genome sequencing approach. Letters in applied microbiology, 66(4), 352-359.

Pascual, D. W., Suo, Z., Cao, L., Avci, R., \& Yang, X. (2013). Attenuating gene expression (AGE) for vaccine development. Virulence, 4(5), 384-390.

Pasetti, M. F., Simon, J. K., Sztein, M. B., \& Levine, M. M. (2011). Immunology of gut mucosal vaccines. Immunological reviews, 239(1), 125-148.

Peterson, A. A., Fesik, S. W., \& McGroarty, E. J. (1987). Decreased binding of antibiotics to lipopolysaccharides from polymyxin-resistant strains of Escherichia coli and Salmonella typhimurium. Antimicrobial agents and chemotherapy, 31(2), 230-237.

Reuven, N. B., Arad, G., Maor-Shoshani, A., \& Livneh, Z. (1999). The mutagenesis protein UmuC is a DNA polymerase activated by UmuD', RecA, and SSB and is specialized for translesion replication. Journal of Biological Chemistry, 274(45), 31763-31766.

Roberts RJ., Vincze T., Posfai J., Macelis D. 2015. REBASE-a database for DNA restriction and modification: enzymes, genes and genomes. Nucleic Acids Research 43:D298-D299. DOI: 10.1093/nar/gku1046.

Rychlik, I., Gregorova, D., \& Hradecka, H. (2006). Distribution and function of plasmids in Salmonella enterica. Veterinary microbiology, 112(1), 1-10.

Sandegren, L., Linkevicius, M., Lytsy, B., Melhus, Å., \& Andersson, D. I. (2011). Transfer of an Escherichia coli ST131 multiresistance cassette has created a Klebsiella pneumoniae-specific plasmid associated with a major nosocomial outbreak. Journal of Antimicrobial Chemotherapy, 67(1), 74-83.

Santos, R. L., Tsolis, R. M., Bäumler, A. J., \& Adams, L. G. (2003). Pathogenesis of Salmonella-induced enteritis. Brazilian Journal of Medical and Biological Research, 36(1), 03-12.

Shariat, N., Timme, R. E., Pettengill, J. B., Barrangou, R., \& Dudley, E. G. (2015). Characterization and evolution of Salmonella CRISPR-Cas systems. Microbiology, 161, 374-386. 
Stubbings, W., Bostock, J., Ingham, E., \& Chopra, I. (2005). Deletion of the multiple-drug efflux pump AcrAB in Escherichia coli prolongs the postantibiotic effect. Antimicrobial agents and chemotherapy, 49(3), 1206-1208.

Summers, A. O. (2002). Generally overlooked fundamentals of bacterial genetics and ecology. Clinical Infectious Diseases, 34(Supplement_3), S85-S92.

Sumrall, E. T., Gallo, E. B., Aboderin, A. O., Lamikanra, A., \& Okeke, I. N. (2014). Dissemination of the transmissible quinolone-resistance gene qnrS1 by IncX plasmids in Nigeria. PLoS One, 9(10), e110279.

Tennant, S. M., \& Levine, M. M. (2015). Live attenuated vaccines for invasive Salmonella infections. Vaccine, 33, C36-C41.

Thanassi, D. G., Bliska, J. B., \& Christie, P. J. (2012). Surface organelles assembled by secretion systems of Gram-negative bacteria: diversity in structure and function. FEMS microbiology reviews, 36(6), 10461082.

Travers, K., \& Michael, B. (2002). Morbidity of infections caused by antimicrobial-resistant bacteria. Clinical Infectious Diseases, 34(Supplement_3), S131-S134.

Vaara, M. (1993). Antibiotic-supersusceptible mutants of Escherichia coli and Salmonella typhimurium. Antimicrobial agents and chemotherapy, 37(11), 2255.

Van, T. T. H., Moutafis, G., Istivan, T., Tran, L. T., \& Coloe, P. J. (2007). Detection of Salmonella spp. in retail raw food samples from Vietnam and characterization of their antibiotic resistance. Appl. Environ. Microbiol., 73(21), 6885-6890.

Wang-Kan, X., Blair, J. M., Chirullo, B., Betts, J., La Ragione, R. M., Ivens, A., .. \& Piddock, L. J. (2017). Lack of AcrB efflux function confers loss of virulence on Salmonella enterica serovar Typhimurium. MBio, 8(4), e00968-17.

Weening EH., Barker JD., Laarakker MC., Humphries AD., Tsolis RM., Bäumler AJ. 2005. The Salmonella enterica serotype typhimurium Ipf, bcf, stb, stc, std, and sth fimbrial operons are required for intestinal persistence in mice. Infection and Immunity 73:3358-3366. DOI: 10.1128/IAI.73.6.3358-3366.2005.

World Health Organization. Management of Substance Abuse Unit. (2014). Global status report on alcohol and health, 2014. World Health Organization.

Yoon, H., McDermott, J. E., Porwollik, S., McClelland, M., \& Heffron, F. (2009). Coordinated regulation of virulence during systemic infection of Salmonella enterica serovar Typhimurium. PLoS pathogens, 5(2), e1000306.

Yue, M., Rankin, S. C., Blanchet, R. T., Nulton, J. D., Edwards, R. A., \& Schifferli, D. M. (2012). Diversification of the Salmonella fimbriae: a model of macro-and microevolution. PloS one, 7(6), e38596. 
Zhou,Y., Liang,Y., Lynch,K.H., Dennis,J.J. and Wishart,D.S. (2011) PHAST: a fast phage search tool. Nucleic Acids Res., 39, W347-W352.

Zgurskaya, H.I., and Nikaido, H. (2000) Multidrug resistance mechanisms: drug efflux across two membranes. Mol Microbio/ 37: 219-225.

\section{Table}

Table 3: Antimicrobial resistance and regulatory genes identified in $S$. Typhimurium UPM 260, LT2, and I 4,[5],12:i:- 


\begin{tabular}{|c|c|c|c|}
\hline Resistance determinants & UPM 260 & $\begin{array}{c}\text { LT2 } \\
\text { (McClelland, 2001) }\end{array}$ & $\begin{array}{c}\text { S. I 4,[5].12:i:- } \\
\text { (Ngoi et. al., 2018) }\end{array}$ \\
\hline AAC(3)-IIa & & & + \\
\hline AAC(6')-Iaa & + & + & + \\
\hline AcrAB & + & + & + \\
\hline AcrD & & & + \\
\hline AcrEF & & & + \\
\hline AdeF & + & & \\
\hline APH(3’)-Ia & + & & \\
\hline APH(3")-Ib (StrA) & + & & \\
\hline APH(6)-Id (StrB) & + & & \\
\hline BacA & + & + & + \\
\hline BaeR & + & + & + \\
\hline $\mathrm{CpxA}$ & + & + & + \\
\hline $\mathrm{CRP}$ & + & + & + \\
\hline EmrD & & & + \\
\hline EmrRB & + & + & + \\
\hline GlpT variant & + & + & + \\
\hline Gols & + & + & \\
\hline $\mathrm{H}-\mathrm{NS}$ & + & + & + \\
\hline KdpE & + & + & + \\
\hline MacAB & & & + \\
\hline MarA & + & + & + \\
\hline MarR & + & + & \\
\hline MdfA & + & & + \\
\hline MdsAC & + & + & \\
\hline MdtK & + & + & + \\
\hline MsbA & + & + & + \\
\hline
\end{tabular}


MsrB

NfsA

$+\quad+$

PatA

$+$

PBP3

$+\quad+$

Pmr operon

$+$

$+$

$+$

QnrS1

$+$

RamA

$+$

RobA

$+$

SdiA

$+$

$+$

$+$

SoxRS

$+\quad+$

Sul2

Bla $_{\text {TEM-1 }}$

$+$

TetA

TetB

$+$

TolC

$+$

$+$

UhpT variant

$+$

$+$

$+$

VgaC

$+$

\section{Declarations}

\section{Acknowledgements}

We thank Nurul Asyifah Mustapha for the tremendous help with the sample preparation. We thank Khalidah Syahirah Ashari for bioinformatics support. We thank each and every one who has contributed to this study.

\section{Ethics approval and consent to participate}

Not applicable

\section{Consent for publication}

Not applicable 


\section{Availability of data and materials}

The annotated draft genome of S. Typhimurium UPM 260 has been deposited in the European Nucleotide Archive with accession number GCA_902153325.1.

https://www.ebi.ac.uk/ena/browser/view/GCA_902153325.1

\section{Competing interests}

The authors declare that they have no competing interests.

\section{Funding}

The study was supported by the FRGS Grant No. FRGS/1/2017/STG05/UPM/02/18 from the Ministry of Higher Education, Government of Malaysia. The funding institution had no direct role in study design, sample collection, analysis, interpretation of data, nor in manuscript writing. Annual reports were submitted to the funding institution tracking the progress of the project.

\section{Authors' contributions}

NSR, NMI, ARO, MHB and Al conceived and designed the study, interpreted the data and wrote and reviewed the manuscript. MHB collected and isolated the strain. NSR operated the study and conducted the objective pathway analyses for WGS data. All authors read and approved the final manuscript and take full responsibility the content and agree to be accountable for all aspects of the work in terms of accuracy or integrity.

\section{Figures}




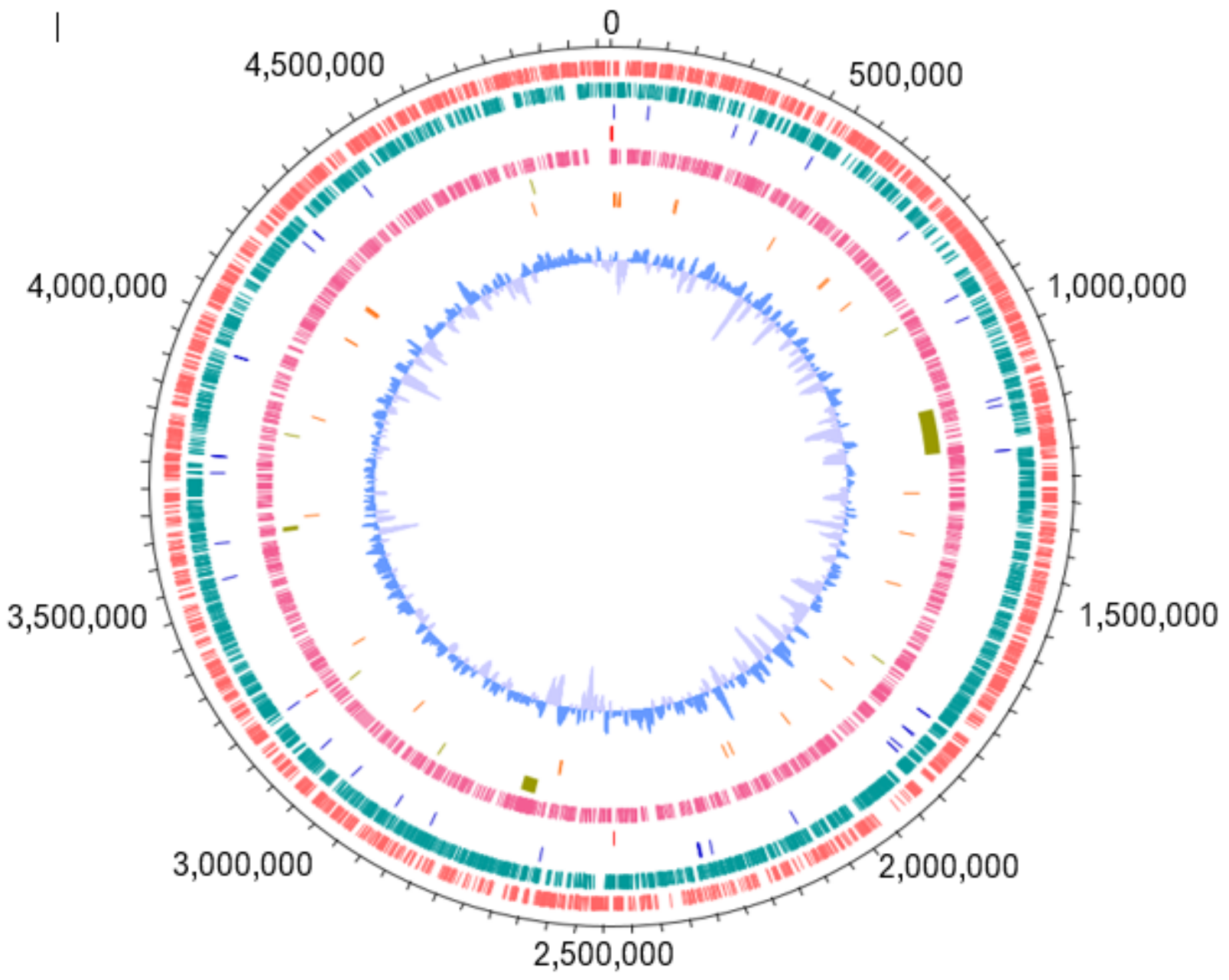

\section{Figure 1}

Circular representation of S. Typhimurium UPM 260 draft genome Tracks from the outermost are as follows: Forward CDS, reverse CDS, tRNA, rRNA, virulence factors, SPIs and antimicrobial resistance genes. The two inner tracks are $\mathrm{G}+\mathrm{C}$ content and GC bias (innermost track). The circular map was generated by using DNAPlotter (Carver et al., 2009). 

A)
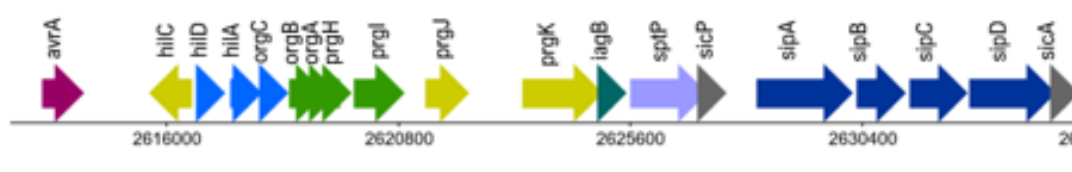
${ }_{2635200}^{5}$
B)

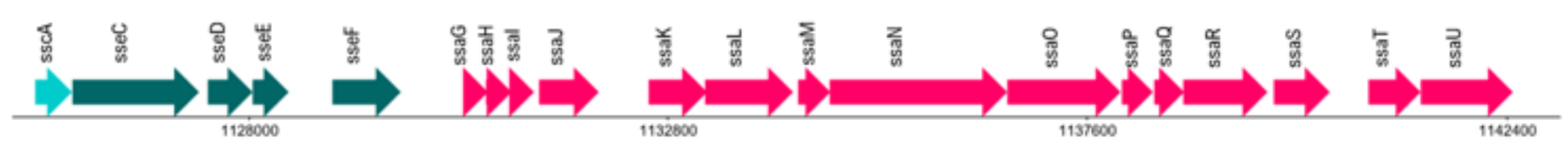
C) $\underset{3562400}{\stackrel{\text { misL }}{\longrightarrow} \underset{3647200}{\mathrm{mgtB}}}$

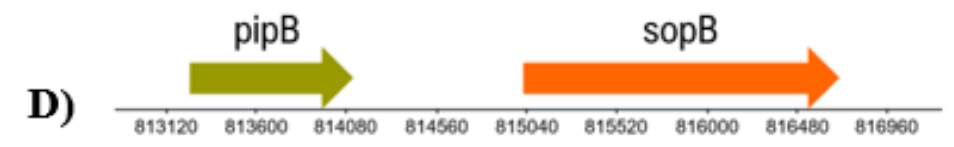

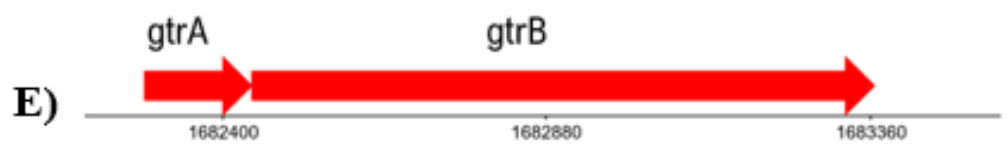

\section{Figure 2}

Genetic organization of virulence factors in (A) SPI-1, (B) SPI-2, (C) SPI-3, (D) SPI-5, (E) SPI-13 